\title{
Influence of coronal mass ejections on parameters of high-speed solar wind: a case study
}

\author{
Yulia Shugay $^{1, *}$, Vladimir Slemzin ${ }^{2}$, Denis Rodkin ${ }^{2}$, Yuri Yermolaev ${ }^{3}$ and Igor Veselovsky ${ }^{1,3}$ \\ ${ }^{1}$ Skobeltsyn Institute of Nuclear Physics, Lomonosov Moscow State University, Moscow, Russia \\ 2 P.N. Lebedev Physical Institute (LPI), Moscow, Russia \\ ${ }^{3}$ Space Research Institute (IKI), Russian Academy of Sciences, Moscow, Russia
}

Received 14 June 2017 / Accepted 21 February 2018

\begin{abstract}
We investigate the case of disagreement between predicted and observed in-situ parameters of the recurrent high-speed solar wind streams (HSSs) existing for Carrington rotation (CR) 2118 (December 2011) in comparison with CRs 2117 and 2119. The HSSs originated at the Sun from a recurrent polar coronal hole $(\mathrm{CH})$ expanding to mid-latitudes, and its area in the central part of the solar disk increased with the rotation number. This part of the $\mathrm{CH}$ was responsible for the equatorial flank of the HSS directed to the Earth. The time and speed of arrival for this part of the HSS to the Earth were predicted by the hierarchical empirical model based on EUV-imaging and the Wang-Sheeley-Arge ENLIL semi-empirical model. The predicted parameters were compared with those measured in-situ. It was found, that for CR 2117 and CR 2119 , the predicted HSS speed values agreed with the measured ones within the typical accuracy of $\pm 100 \mathrm{~km} \mathrm{~s}^{-1}$. During CR 2118, the measured speed was on $217 \mathrm{~km} \mathrm{~s}^{-1}$ less than the value predicted in accordance with the increased area of the $\mathrm{CH}$. We suppose that at CR 2118, the HSS overtook and interacted with complex ejecta formed from three merged coronal mass ejections (CMEs) with a mean speed about $400 \mathrm{~km} \mathrm{~s}^{-1}$. According to simulations of the Drag-based model, this complex ejecta might be created by several CMEs starting from the Sun in the period between 25 and 27 December 2011 and arriving to the Earth simultaneously with the HSS. Due to its higher density and magnetic field strength, the complex ejecta became an obstacle for the equatorial flank of the HSS and slowed it down. During CR 2117 and CR 2119, the CMEs appeared before the arrival of the HSSs, so the CMEs did not influence on the HSSs kinematics.
\end{abstract}

Keywords: solar wind prediction models / high-speed solar wind streams / coronal holes / coronal mass ejections / interplanetary coronal mass ejections / HSS-CME interaction

\section{Introduction}

Among various solar wind disturbances, high speed streams (HSSs) of solar wind originating from coronal holes (CHs) represent one of the main drivers of geomagnetic storms (Tsurutani et al., 1995, 2006; Yermolaev et al., 2005, 2009; Echer et al., 2011a,b; Myagkova et al., 2013). Numerous studies have shown that geoeffectiveness of HSSs is determined by their speed, duration and magnetic field parameters. It is well established that there is a direct connection between the passage of the low and mid-latitude parts of CHs across the solar disk and the recurrent high-speed streams recorded at the Earth orbit (Nolte et al., 1976; Harvey \& Sheeley, 1978; Wang \& Sheeley, 1990).

During propagation in the solar corona and heliosphere, HSSs can interact with the ambient slow solar wind and other

*Corresponding author: jshugai@srd.sinp.msu.ru streams like coronal mass ejections (CMEs). Interaction of recurrent HSSs with slow solar wind in the heliosphere can generate corotating interaction regions (CIRs), which often produce moderate geomagnetic storms (Alves et al., 2006). Interaction of HSSs with CMEs is of particular interest for the space weather forecasting because it can strengthen or weaken geoeffectiveness of the event. Influence of different coronal structures as streamers, $\mathrm{CHs}$, other CMEs, and the ambient solar wind on propagation of CMEs are the subject of many studies (see, for instance, papers by Gopalswamy et al., 2004; Mishra et al., 2015; Möstl et al., 2015 and references therein). Authors of several works (Gopalswamy et al., 2009; Mohamed et al., 2012; Nieves-Chinchilla et al., 2013) showed that presence of $\mathrm{CHs}$ can change the CME trajectory. In particular, polar $\mathrm{CHs}$ can deflect $\mathrm{CMEs}$ to the equator. Behannon et al. (1991); Burlaga et al. (2002, 2003); Rouillard et al. (2010) discussed existence of compound structures in the solar wind - merged interaction regions (MIRs) formed by the CMEHSS interaction and observed in-situ in the solar wind. The MIRs are relatively large structures with the radial extent 
$\approx 2 / 3 \mathrm{AU}$ and $3 / 4 \mathrm{AU}$ and may include a complex ejecta, a magnetic cloud, shocks, a corotating stream and a heliospheric plasma sheet. Fenrich \& Luhmann (1998) found that a fast solar wind stream could compress the preceding magnetic cloud, which results in increase of the plasma density and magnetic field strength providing a significant effect upon geomagnetic storm intensity if the magnetic cloud is of N-S polarity. However, the above mentioned papers did not consider influence of the CME-HSS interaction on the observed in-situ parameters of the HSS.

Currently several models are used for prediction of the solar wind parameters in the near-Earth space and beyond, which consider HSSs and CMEs as independent features. The quality of these models is evaluated by the disagreement between prediction and measurements of different solar wind parameters, among which the most important are the arrival time and speed at $1 \mathrm{AU}$ (Zhao \& Dryer, 2014; Jian et al., 2015; Reiss et al., 2016). The semi-empirical Wang-Sheeley-Arge model (WSA: Wang \& Sheeley, 1990; Arge \& Pizzo, 2000) based on the magnetic field expansion factor, the advanced Magnetohydrodynamic-based WSA-ENLIL prediction model (Odstrčil \& Pizzo, 1999) and the empirical models based on the EUV-imaging (e.g. Vršnak et al., 2007; Krista \& Gallagher, 2009; Shugay et al., 2011; Rotter et al., 2015) are most often used in operational practice. All types of the models use position and geometry of $\mathrm{CHs}$ for simulation of quasistationary streams of HSSs, but the EUV-imaging models are better following the short-time variations in the $\mathrm{CH}$ geometry, whereas the WSA and WSA-ENLIL models more effectively follow the global magnetic field configuration of the Sun.

CMEs originated at the Sun propagate through the heliosphere as expanding plasma structures reaching the Earth as interplanetary coronal mass ejections (ICMEs). Commonly two types of the models are often used for prediction of the arrival time and speed of ICMEs based on the near-Sun coronagraphic observations. Among the kinematic empirical models, the most advanced Drag-based model (DBM: Vršnak et al., 2013) consider that propagation of a $\mathrm{CME}$ in the heliosphere is governed by its interaction with the ambient solar wind depending on their relative speeds. A CME propagates with deceleration, if the stream velocity is higher than that of the ambient wind, or with acceleration in the opposite case. A modification of the WSAENLIL model, the WSA-ENLIL Cone model (Mays et al., 2015), provides simulation of a CME propagation in the heliosphere using information about initial parameters of CME from coronagraphic observations. This model describes expansion of the CME plasma in the heliosphere providing its speed and density as functions of time and distance in three dimensions, but likewise the kinematic models, it does not take into account probable magnetic interaction between HSS and $\mathrm{CME}$ and its influence on the solar wind parameters in the near-Earth space.

Reiss et al. (2016) compared effectiveness of the empirical solar wind forecast (ESWF) model based on a relation between the areas of CHs as observed in EUV and the solar wind speed at $1 \mathrm{AU}$, and the WSA model. They found that $70 \%$ of the associated high-speed enhancements are correctly predicted by the ESWF model within $\pm 100 \mathrm{~km} \mathrm{~s}^{-1}$ and $52 \%$ are correctly predicted within $\pm 50 \mathrm{~km} \mathrm{~s}^{-1}$ for the period 2011-2014. In comparison, the WSA model correctly predicts $78 \%$ of the high-speed enhancements with the accuracy of $\pm 100 \mathrm{~km} \mathrm{~s}^{-1}$ and $49 \%-$ within $\pm 50 \mathrm{~km} \mathrm{~s}^{-1}$. The hierarchical empirical prediction model used in this work (Shugay et al., 2011, hereafter - the HE model) is similar to the ESWF tool in the principle of operation and has similar accuracy.

However, in some cases the disagreement between prediction and measurements surpasses the typical inaccuracy for mentioned models $\sim 100 \mathrm{~km} \mathrm{~s}^{-1}$ in two or more times. To improve the quality of the forecast, it is important to identify the causes of such strong disagreement. In particular, the HSS propagation anomalies can be caused by residual transient distortion of the background solar wind after passing a CME (Temmer et al., 2017). The authors investigated the impact of the detected in-situ ICMEs to the speed of the background solar wind predicted by different solar wind models (ESWF, WSA, persistence model) for the period 2011-2015. The observed solar wind speed within the ICME interval showed an increase of $18-32 \%$ above the expected background, and during the period of two days after the ICME displayed an increase of $9-24 \%$. The total duration of enhanced deviations lasted over about three and up to six days after the ICME start, which is much longer than the average duration of an ICME disturbance itself. The studied solar wind disturbances surely should influence on the HSS propagation, although the authors did not mention it. One can suppose, that the largest disagreement between the predicted and measured arrival time and speed of a HSS may be caused by its direct interaction with a CME (or several CMEs) in the heliosphere.

In this work, we consider predictions of three HSSs occurred in the consecutive Carrington rotations (CRs) during November 2011-February 2012 (CRs 2117-2119), when the same recurrent polar $\mathrm{CH}$ expanded to middle latitudes crossed the central meridian. In our recent publication (Shugay et al., 2017) we mentioned this case as an example of strong disagreement between the HSS forecast and measurements, and here we give a detailed analysis of the events. According to the predictions by various models, the HSSs should be observed near the Earth in all rotations with the speed increasing due to the expanding middle- and low-latitude area of the $\mathrm{CH}$. We consider disagreement between predictions and observations of the HSSs, which can be consequences of interaction between HSSs and cotemporal Earth-directed CMEs in the heliosphere. Our consideration is based on predictions by the HE model. We refer to simulations by the WSA-ENLIL Cone model only to demonstrate that the result is model independent.

\section{Methods}

\subsection{Prediction of solar wind phenomena by the HE model}

To predict arrival of HSS and transient CME streams to the Earth, one needs first to determine the sources of these streams, which can be identified by specific signatures. HSSs originate in $\mathrm{CHs}$, which can be identified as large regions of open magnetic field lines or seen in the EUV wavelength range (typically, in the $19.3 \mathrm{~nm}$ line) as wide dark areas (Fig. 1, the upper panel). The procedure of the $\mathrm{CH}$ identification applied in the HE model has been described in Shugay et al. (2011, 2014); Slemzin \& Shugai (2015). 

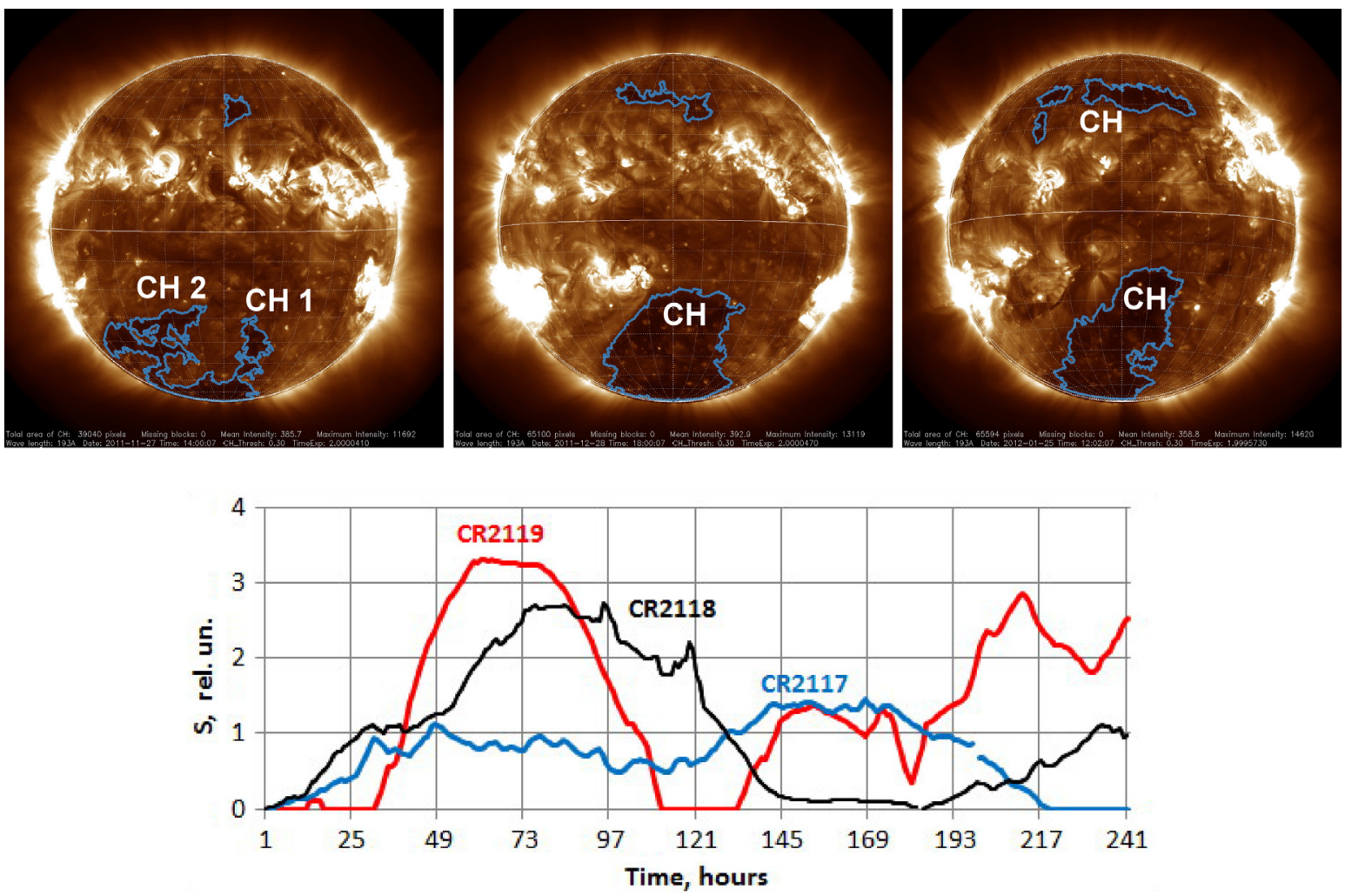

Fig. 1. Top: images of the Sun at wavelength $19.3 \mathrm{~nm}$ from SDO/AIA for CR 2117, 2118 and 2119 (from left to right on 27 November 2011,28 December 2011 and 25 January 2012). Bottom: temporal variation of the $\mathrm{CH}$ area (in relative units) for three CRs. The abscissa is relative time in hours.

To predict the arrival time and velocity of HSSs at $1 \mathrm{AU}$, we used as the initial data the EUV images in $19.3 \mathrm{~nm}$ band from the Advanced Imaging Assembly telescope on the Solar Dynamic Observatory (SDO/AIA) (Lemen et al., 2012) taken in the standard FITS-format, the sampling rate being one image per hour. In our model, we identify $\mathrm{CHs}$ by the intensity below the threshold value, which is calculated as $I_{\text {mean }} \cdot k$, where $I_{\text {mean }}$ is the average intensity of the solar disk without taking into account the limb brightening, and $k$ is the empirical threshold coefficient, different for various wave length and spacecraft (Shugay et al., 2011). The regions of decreased intensity in the images of the Sun in the EUV wavelength range correspond not only to the regions of $\mathrm{CHs}$, but also include the filament channels (FC). We excluded FCs by analysis of magnetic field distribution in the dimmed regions, which is different in CHs and FCs (Scholl \& Habbal, 2008). To separate the CHs from FCs, we consider the data on the radial component of the photospheric magnetic field measured by the Helioseismic and Magnetic Imager on SDO (Hurlburt et al., 2012).

The solar wind sources positioned in the central part of the solar disk produce a measurable contribution to solar wind near the Earth. Therefore, we calculate the area of $\mathrm{CHs}$ within the source region confined by $\pm 20^{\circ}$ longitude from the central meridian of the Sun and by $\pm 50^{\circ}$ latitude from the solar equator. The HSS velocity is calculated as a linear function of the $\mathrm{CH}$ area (Slemzin \& Shugai, 2015). We used this model for association of the passage of $\mathrm{CH}$ over the solar disk with the arrival of the HSS at $1 \mathrm{AU}$. The velocity of the solar wind as a function of time is calculated as follows:

$$
V\left(S_{i}, t\right)=V_{\min }+A_{i} S_{i}\left(t_{i_{0}}\right),
$$

where $S_{i}\left(t_{i_{0}}\right)$ is the $\mathrm{CH}$ relative area taken in the selected range in latitude and longitude at the time $t_{i_{0}}$ at the wavelength $\lambda_{i}$, $V_{\min }$ is the minimum (background) solar wind velocity, $A_{i}$ is a parameter depending on the wavelength $\lambda_{i}$, and $t$ is the time of the HSS arrival to the near-Earth orbit according to the velocity prediction. The HE model calculates the HSS arrival time from the speed temporal profile with the transition time determined under the assumption that the stream speed in the heliosphere is constant (the ballistic approximation). The prediction of the HSS arrival time and speed at $1 \mathrm{AU}$ using the above described model based on the SDO/AIA data is available in real time on the site of Skobeltsyn Institute of Nuclear Physics of Moscow State University (SINP/MSU) ${ }^{1}$.

The accuracy of HE model was tested at the period January 2011-February 2012. The root-mean-square error (RMSE) of the forecast is given by the relation:

$$
\sigma_{f}=\sqrt{\frac{1}{n} \Sigma\left(V_{o}-V_{f}\right)^{2}}
$$

where $n$ - total number of observed and predicted pairs, $V_{0}-$ the observed HSS speed and $V_{f}-$ the forecasted speed. For the mentioned period, the HE model has RMSE $\sigma_{f}=105 \mathrm{~km} \mathrm{~s}^{-1}$,

\footnotetext{
$\overline{1} \mathrm{http}: / /$ swx.sinp.msu.ru/models/solar_wind.php?gcm=1\&lang=en
} 
and the correlation coefficient between the observed and predicted values equals to 0.45 . It should be mentioned that these estimations were obtained from the difference between the predicted HSS speed and the real speed of the solar wind including CMEs. The HE model correctly predicted $73 \%$ of all solar wind streams with the speed more than $400 \mathrm{~km} \mathrm{~s}^{-1}$ with deviation from observations within the range $\pm 100 \mathrm{~km} \mathrm{~s}^{-1}$. For these simulations we used the speed of the background solar wind $\left(V_{\min }\right)$ equal to $300 \mathrm{~km} \mathrm{~s}^{-1}$, as the most probable value of the minimum speed of the solar wind during the period 2011-2012.

CMEs originate due to spontaneous solar activity phenomena associated with X-ray flares and flux rope ejections in active regions (ARs), or due to gradual filament/prominence eruptions without flares outside ARs. Such signatures as fast loop expansion, dimmings, coronal waves or post-eruption arcades can localize their solar sources. To link ICMEs with CMEs directed to the Earth, we used the data from the COR2 coronagraphs on two Solar Terrestrial Relations Observatories (STEREO-A and B, Howard et al., 2008), presented in the Sun-Earth Connection Coronal and Heliospheric Investigation instrument database ${ }^{2}$ and C2 instrument of Large Angle and Spectrotrometric Coronagraph (LASCO: Brueckner et al., 1995) onboard the Solar and Heliospheric Observatory (SOHO: Domingo et al., 1995), presented in the Coordinated Data Analysis Workshops (CDAW) CME list ${ }^{3}$. To study solar origins of the identified CMEs we used EUV images from the SDO/AIA telescope ${ }^{4}$ and the Solar Demon database ${ }^{5}$ of the event detection by flares, dimmings and EUV waves. For calculations of the CME arrival time we used two heliospheric CME-propagation models: $\mathrm{DBM}^{6}$ (Vršnak et al., 2013) and the WSA-ENLIL Cone model (Odstrčil \& Pizzo, 1999; Pizzo et al., 2011) ${ }^{7}$, both available online. Below, we will denote the data obtained from STEREO-A as the STA data and those from STEREO-B as STB data. In the period under study STEREO$A$ and $B$ were located near the ecliptic plane at the angles 106$108^{\circ}$ and $(-105)$ to $(-115)^{\circ}$ relative to the Sun-Earth line.

\subsection{Identification of the solar wind phenomena in-situ}

Solar wind phenomena are typically identified using the standard criteria for the solar wind parameters (Sheeley \& Harvey, 1981; Burlaga, 1991; Lepping et al., 2003; Zurbuchen \& Richardson, 2006; Yermolaev et al., 2009; Borovsky \& Denton, 2010; Mitsakou \& Moussas, 2014; Prise et al., 2015). The solar wind features observed in connection with the HSS propagation are CIRs and HSSs (body): CIR is an interaction region between the slow and fast solar wind streams and characterized by an increase and then a decrease in the proton density, an increase in the velocity and kinetic temperature of the protons, an increase in the strength of the interplanetary magnetic field; HSS has the bulk speed $V>450 \mathrm{~km} \mathrm{~s}^{-1}$ (in the rise of Cycle $24 V>400 \mathrm{~km} \mathrm{~s}^{-1}$, Rod'kin et al., 2016) and low values of the proton density and magnetic field. Propagation of

\footnotetext{
2 http://helio.gmu.edu/seeds/secchi.php

3 http://cdaw.gsfc.nasa.gov/CMElist/index.html

$4 \mathrm{http}: / /$ sdo.gsfc.nasa.gov

$5 \mathrm{http}: / /$ solardemon.oma.be/

6 http://oh.geof.unizg.hr/DBM/dbm.php

${ }^{7} \mathrm{http}: / /$ helioweather.net
}

a CME can cause the signatures of such phenomena as interplanetary shock (IS), sheath (SH), and ICME. Interplanetary shock is a sudden increase of the solar wind speed on more than $20 \mathrm{~km} \mathrm{~s}^{-1}$ accompanied with jumps in the plasma and magnetic field parameters; $\mathrm{SH}$ is a compression region before fast ICME an enhanced proton temperature, density and beta parameter. Numerical criteria and procedure of identification are described in details by Yermolaev et al. (2009), the catalog of the identified solar wind phenomena for 1976-2016 is presented at the web-site of Space Research Institute, Moscow (IKI solar wind database) ${ }^{8}$.

By analysis of the data of the OMNI dataset ${ }^{9}$ for the period 1976-2000 and using the method of the double superposed epoch analysis for large numbers of events (Yermolaev et al., 2010), we classified and parameterized temporal sequences of phenomena in assumption of absence of interaction with other streams (Yermolaev et al., 2015). As a result, the averaged temporal profiles for the main parameters of solar wind and IMF have been obtained. Similar methods of the solar wind profile analysis were effectively used in the papers by Yokoyama \& Kamide (1997); Lepping et al. (2003); Kilpua et al. (2015). It should be noted that this method does not take into account the situations when different solar wind phenomena follow one after another and interact in the heliosphere. Thus, difference between measured and standard parameters of the classified phenomena can indicate such interaction in each particular case.

We analyzed solar wind parameters using the data from the Advanced Composition Explorer (ACE) and the WIND spacecraft for the period November 2011-February 2012. The hourly averaged values of the IMF and the solar wind parameters were taken from the instruments Magnetic Field Experiment (MAG: Smith et al., 1998) and Solar Wind Electron, Proton, and Alpha Monitor (SWEPAM: McComas et al., 1998) on ACE and the Solar Wind Experiment on WIND (SWE: Gloeckler et al., 1995). We used the ACE hourly averaged data (proton speed, magnetic field, $\mathrm{He} / \mathrm{H}$ ratio) supplemented by the WIND hourly data (proton density, proton temperature) in the ACE data gaps. The beta parameter was calculated from the measured proton density, temperature, and magnetic field strength.

\section{Results and discussion}

In the next sections, we consistently describe observations of Sun, measurements of the solar wind parameters at $1 \mathrm{AU}$ and their comparison with the model predictions in the period from the end of November 2011 to the beginning of February 2012 (CRs 2117-2119).

\subsection{Prediction of HSSs and comparison with in-situ observations}

During CRs 2117-2119, the potential solar sources of HSS were the large recurrent southern polar $\mathrm{CH}$ extended to the middle latitudes and, with smaller contribution, the growing high-latitude northern polar $\mathrm{CH}$. Figure 1 (upper panel) shows

\footnotetext{
8 ftp://ftp.iki.rssi.ru/pub/omni/catalog/

${ }^{9}$ http://omniweb.gsfc.nasa.gov
} 
Table 1. Times of maximum $\mathrm{CH}$ area, predicted and observed parameters of HSSs.

\begin{tabular}{|c|c|c|c|c|c|}
\hline $\mathrm{CR}$ & $\begin{array}{l}\text { Date and time (UT) } \\
\text { when } \mathrm{CH} \text { has the max } \\
\text { area within } \pm 50^{\circ} \text { latitude } \\
\text { and } \pm 20^{\circ} \text { longitude }\end{array}$ & $\begin{array}{l}\text { Predicted HSS arrival } \\
\text { data and time, UT (start/end) }\end{array}$ & $\begin{array}{l}\text { Observed HSS arrival } \\
\text { data and time, UT } \\
\text { (start/end) }\end{array}$ & $\begin{array}{l}\text { Predicted by the } \\
\text { HE model HSS max } \\
\text { speed, } \mathrm{km} \mathrm{s}^{-1}\end{array}$ & $\begin{array}{l}\text { Observed HSS } \\
\text { max speed, } \mathrm{km} \mathrm{s}^{-1}\end{array}$ \\
\hline \multirow[t]{2}{*}{2117} & (1) 25 Nov. $2011,23: 00$ & $\begin{array}{l}29 \text { Nov. } 2011,12: 00 / \\
3 \text { Dec. } 2011,10: 00\end{array}$ & $\begin{array}{l}30 \text { Nov. } 2011,18: 00 / \\
2 \text { Dec. } 2011,10: 00\end{array}$ & 425 & 482 \\
\hline & (2) 30 Nov. $2011,06: 00$ & $\begin{array}{l}3 \text { Dec. } 2011,11: 00 / \\
7 \text { Dec. } 2011,00: 00\end{array}$ & $\begin{array}{l}3 \text { Dec. } 2011,13: 00 / \\
7 \text { Dec. } 2011,00: 00\end{array}$ & 457 & 469 \\
\hline 2118 & 26 Dec. $2011,11: 00$ & $\begin{array}{l}30 \text { Dec. } 2011,06: 00 / \\
4 \text { Jan. } 2012,19: 00\end{array}$ & $\begin{array}{l}31 \text { Dec. } 2011,00: 00 / \\
2 \text { Jan. } 2012,03: 00\end{array}$ & 597 & 380 \\
\hline 2119 & 24 Jan. $2012,12: 00$ & $\begin{array}{l}27 \text { Jan. 2012, 05:00/ } \\
31 \text { Jan. 2012, 12:00 }\end{array}$ & $\begin{array}{l}27 \text { Jan. 2012, 07:00/ } \\
30 \text { Jan. 2012, 16:00 }\end{array}$ & 634 & 552 \\
\hline
\end{tabular}

the locations of the CHs in the SDO/AIA $19.3 \mathrm{~nm}$ solar images at CRs 2117-2119. The bottom panel shows the temporal variation of the $\mathrm{CH}$ areas in the source region within the $\pm 20^{\circ}$ longitude and $\pm 50^{\circ}$ latitude. Parameters of the HSS solar sources, predicted by the HE model and observed solar wind speed and the arrival times in UT are listed in Table 1. Figure 2 shows the temporal dependence of the predicted solar wind speed calculated by the HE model and by the WSA-ENLIL Cone model in comparison with the speed measured by ACE. The mid-latitude $\mathrm{CHs}$ produce the solar wind with the moderate speed (typically, $450-550 \mathrm{~km} \mathrm{~s}^{-1}$, in Cycle 24-above $400 \mathrm{~km} \mathrm{~s}^{-1}$ ), low density and the IMF strength in the body of the stream after passage of CIRs.

Figure 1 shows that the southern $\mathrm{CH}$ at $\mathrm{CR} 2117$ extended to the solar equator to $\sim 30^{\circ}$ and at CR 2118 to $25^{\circ}$ of the southern latitude. At $\mathrm{CR} 2117$, the $\mathrm{CH}$ area have two maxima due to two parts of the $\mathrm{CH}$ area that expand below $50^{\circ}$ of the southern latitude (Fig. 1), so it produced two HSSs - HSS 1 and HSS 2 (Tab. 1) with the predicted speeds corresponding to the value of the maximum $\mathrm{CH}$ area. In accordance with the observed geometry of the $\mathrm{CH}$ during all three $\mathrm{CRs}$, the $\mathrm{HE}$ model predicted occurrence of the HSS near the Earth with the speed growing with the rotation number (Tab. 1).

Figure 2 shows the ACE measurements of the solar wind speed for CRs 2117-2119 (the black line) in comparison with predictions by the HE model (the yellow line) and the WSAENLIL Cone model (the green line). In the period from 29 November 2011 to 7 December 2011 (CR 2117), the solar wind speed (black line) has two broad maxima: from 30 November 2011, 18:00 UT to 2 December 2011, 10:00 UT (HSS 1), and from 3 December 2011, 13:00 UT to 7 December 2011, 00:00 UT (HSS 2) associated with two parts of mid-latitude extensions of the southern $\mathrm{CH}$. According to the data in Table 1, the observed maximal values of the solar wind speed agree with predictions by the HE model within the uncertainty less than $60 \mathrm{~km} \mathrm{~s}^{-1}$. The WSA-ENLIL Cone model showed larger disagreement within $\pm 100 \mathrm{~km} \mathrm{~s}^{-1}$ in the period 2-3 December 2011, probably, associated with the erroneous prediction of a CME arrival in the tail of HSS 1.

In the period from 28 December 2011, 06:00 UT to 7 January 2012, 19:00 UT (CR 2118), the HE model predicted the HSS arrival on 30 December 2011 at 06:00 UT with the speed of $597 \mathrm{~km} \mathrm{~s}^{-1}$, but really the solar wind with some signatures of HSS (see in the next Section) was observed starting from 31 December 2011, 00:00 UT, with the speed $380 \mathrm{~km} \mathrm{~s}^{-1}$, that is on $217 \mathrm{~km} \mathrm{~s}^{-1}$ less than it was predicted. Such disagreement between the predicted and measured arrival times and speeds of the HSS sufficiently exceeds the typical uncertainty of the HE model. A decrease of the HSS speed at CR 2118 in comparison with that at CR 2117 cannot be explained by variation of the $\mathrm{CH}$ geometry, because at $\mathrm{CR}$ 2118 the $\mathrm{CH}$ area in the source region increased, and its upper boundary shifted towards the equator.

At CR 2119, according to Figure 1, the boundary of the recurrent $\mathrm{CH}$ was located at $20^{\circ}$ southward from the equator, and its geoeffective area increased in comparison with the preceding rotations due to the part of the northern $\mathrm{CH}$. The HE model predicted appearance of the HSS in the period from 27 January 2012, 05:00 UT to 31 January 2012, 12:00 UT with the maximal speed of $634 \mathrm{~km} \mathrm{~s}^{-1}$. The observed speed of the HSS has reached $550 \mathrm{~km} \mathrm{~s}^{-1}$, which is on $84 \mathrm{~km} \mathrm{~s}^{-1}$ less than the predicted value and lies in the accuracy limits of the HE model.

We suggest that the significant disagreement between prediction and observation of the HSS at CR 2118 might be caused by interaction of the HSS with the CMEs propagated in the heliosphere towards the Earth at the same period. In the next sections, we investigate in detail the solar wind parameters around the periods of the HSS prediction and the solar phenomena, which can influence on the HSS propagation.

\subsection{Solar phenomena and their influence on the HSS parameters}

Table 2 presents information about CMEs started during the period, when the recurrent $\mathrm{CH}$ was present in the source region at the solar disk, and predictions on their arrival to the Earth. These CMEs were selected from the STEREO and LASCO CME databases as it was described in the Section 2. Their arrival time at $1 \mathrm{AU}$ and speed were estimated using DBM (the speed of the background solar wind was taken to be $300 \mathrm{~km} \mathrm{~s}^{-1}$, similar to 

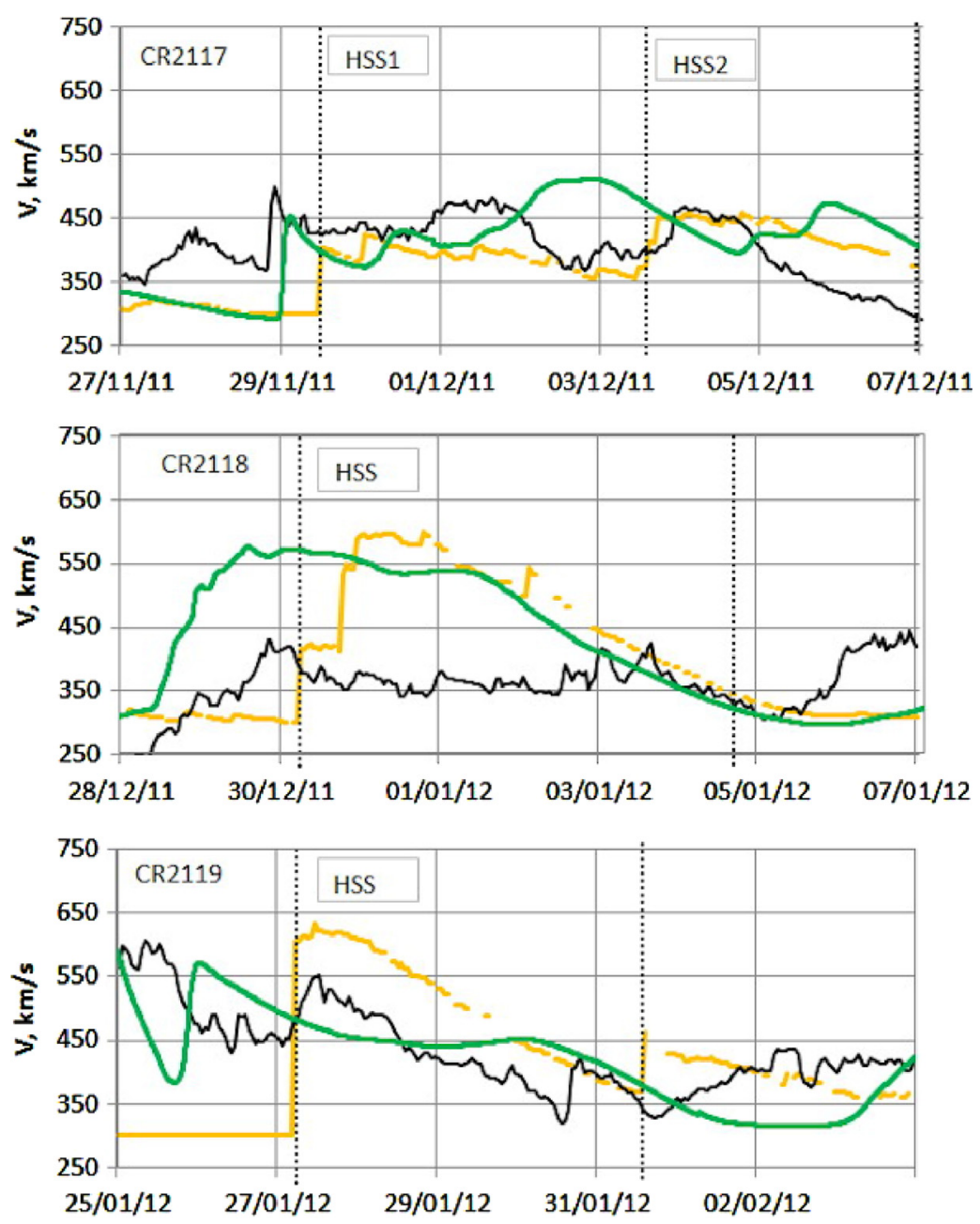

Fig. 2. Data presented from top to bottom for CRs 2117-2119. The proton speed measured by ACE (the black curve), the proton speed calculated by the HE model (the yellow curve) and calculated by the WSA-ENLIL Cone model (the green curve).

that taken for the HSS simulations with the HE model). Figures 3, 5 and 7 demonstrate the solar wind parameters measured in-situ near the periods of the predicted HSS arrivals.

At CR 2117, according to two maxima in the temporal dependence of the $\mathrm{CH}$ area (see Fig. 1), two HSSs-HSS 1 and HSS 2 were formed at the Sun. HSS 1 reached its maximal speed on 25 November 2011, 23:00 UT. The HE model predicted its arrival to the Earth in the period from 29 November 2011, 12:00 UT to 3 December 2011, 10:00 UT with the speed $425 \mathrm{~km} \mathrm{~s}^{-1}$. Nine hours after the $\mathrm{CH}$ areas reached its maximum size, a halo CME 1 started on 26 November 2011, at 08:24(STA)/07:55(STB) UT with the speed $1139 / 821 \mathrm{~km} \mathrm{~s}^{-1}$. DBM (Tab. 2) predicted the arrival of CME 1 on 29 November 2011, 02:00 UT with the speed of $460 \mathrm{~km} \mathrm{~s}^{-1}$.

The solar wind measurements (Fig. 3) show a shock on 28 November 2011, 21:00 UT followed by a SH region of enhanced density, temperature and magnetic field (28 November 2011, 22:00 UT-29 November 2011, 18:00 UT), an ICME from 29 November 2011, 19:00 UT to 30 November 2011, 17:00 UT and the HSS signatures from 30 November 2011, 18:00 UT to 2 December 2011, 10:00 UT. This complex structure represents a MIR, when two or several CMEs merge with HSS (Burlaga et al.,
2003; Rouillard et al., 2010 and references therein). In our case, MIR lasting from 28 November 2011, 22:00 UT to 30 November 2011, 17:00 UT included a shock, a SH, an ICME and CIR followed by the HSS body. The snapshot of the WSA-ENLIL Cone modeling (Fig. 4) shows that CME 1 overtook HSS 1 in the heliosphere and arrived to the Earth practically at the same time and with the same speed.

After HSS 1, the solar wind data contains signatures of the second ICME from 2 December 2011, 11:00 UT with the speed $390 \mathrm{~km} \mathrm{~s}^{-1}$. Most probably, this ICME corresponds to CME 2 started from the Sun on 28 November 2011, 11:24(STA) UT with the speed $322 \mathrm{~km} \mathrm{~s}^{-1}$. According to the DBM simulation, CME 2 was predicted to arrive on 2 December 2011, 19:00 UT, with a speed $320 \mathrm{~km} \mathrm{~s}^{-1}$ that corresponds to the speed of the observed ICME within the DBM accuracy. The $\mathrm{CH}$ corresponding to the HSS 2 reached its maximum size two days later, 30 November 2011, 06:00 UT, and arrived on 3 December 2011, 13:00 UT with the speed $469 \mathrm{~km} \mathrm{~s}^{-1}$. Thus, these streams propagating one after another did not interacted in the heliosphere, so they did not generated a shock and a MIR.

At CR 2118, according to the predictions by the HE model (Tab. 1), the $\mathrm{CH}$ reached its maximum area on 26 December 2011 at 11:00 UT, and the corresponding HSS should arrive to 
Table 2. CMEs and predicted ICME arrival time during CRs 2117-2119.

\begin{tabular}{|c|c|c|c|c|}
\hline $\mathrm{N}$ of $\mathrm{CME}$ & $\begin{array}{l}\text { CME appearance time, } \\
\text { UT (A) STA, (B) STB, } \\
\text { (L) LASCO }\end{array}$ & $V_{C M E}, \mathrm{~km} \mathrm{~s}^{-1}$ & $\begin{array}{l}\text { DBM predicted/measured } \\
\text { time of ICME arrival, UT }\end{array}$ & $\begin{array}{l}\text { Predicted } / \text { measured, } \\
V_{I C M E}, \mathrm{~km} \mathrm{~s}^{-1}\end{array}$ \\
\hline \multirow[t]{3}{*}{1} & (A) 26 Nov. $2011,08: 24$ & 1139 & 29 Nov. 2011, 02:00/ & $460 / 430$ \\
\hline & (B) 26 Nov. $2011,07: 55$ & 821 & 29 Nov. $2011,19: 00$ & - \\
\hline & (L) 26 Nov. $2011,07: 12 \mathrm{H}$ & 858 & - & - \\
\hline 2 & (L) 27 Nov. 2011, 10:36 PH & 322 & 2 Dec. 2011, 11:00 & - \\
\hline \multicolumn{5}{|l|}{ CR 2118} \\
\hline \multirow[t]{4}{*}{1} & (A) 25 Dec. $2011,00: 54$ & 445 & 28 Dec. $2011,13: 00-$ & $390-420 /$ \\
\hline & (B) 25 Dec. $2011,01: 25$ & 545 & 30 Dec. $2011,06: 00 /$ & 411 \\
\hline & (L) 25 Dec. $2011,01: 26 \mathrm{H}$ & 672 & & - \\
\hline & (L) 26 Dec. $2011,11: 48 \mathrm{PH}$ & 884 & - & - \\
\hline \multirow[t]{2}{*}{2} & (A) 29 Dec. $2011,17: 24$ & 555 & 2 Jan. 2012, 11:00/ & $400 / 358$ \\
\hline & (L) 29 Dec. 2011, 16:24 PH & 704 & 2 Jan. 2012, 04:00 & - \\
\hline \multicolumn{5}{|l|}{ CR 2119} \\
\hline \multirow[t]{3}{*}{1} & (A) 23 Jan. 2012, 04:24 & 1217 & 25 Jan. $2012,17: 00 /$ & $480 / 461$ \\
\hline & (B) 23 Jan. $2012,03: 25$ & 976 & 26 Jan. 2012, 14:00 & - \\
\hline & (L) 23 Jan. $2012,04: 00 \mathrm{H}$ & 2065 & - & - \\
\hline
\end{tabular}

the Earth on 30 December 2011, 06:00 UT with the speed of $597 \mathrm{~km} \mathrm{~s}^{-1}$. The ACE data in Figure 5 show that during the period from 28 December 2011, 03:00 UT and 30 December 2011, 06:00 UT, there was a period of strong disturbances in the solar wind, marked in Figure 5 by the red horizontal bar, which included large enhancements in the proton density, speed, temperature and magnetic field strength. After these disturbances, until 2 January 2012, 04:00 UT, there was a short ICME, marked by the green bar, followed by the short-time enhancement in density, temperature and magnetic field strength, which may be identified as a stream interface region. From 31 December 2011, 00:00 UT to 2 January 2011, 04:00 UT there is a period of the turbulent solar wind, in which such plasma parameters, as proton temperature, magnetic field and beta, intermittently displayed signatures of HSS and ICME. In Figure 5, this period of the solar wind is marked by the blue dashed bar. As it was noted in Section 3.1, during this period the maximum speed of the HSS did not exceed $380 \mathrm{~km} \mathrm{~s}^{-1}$, which is $217 \mathrm{~km} \mathrm{~s}^{-1}$ less than the predicted one.

Such feature, which includes the complex ejecta, the ICME, CIR and MIR like that identified at CR 2117, but with the more pronounced signatures. In particular, the proton density reached $47 \mathrm{~cm}^{-3}$ versus $20 \mathrm{~cm}^{-3}$ in the previous case, the proton temperature $-1 \times 10^{5} \mathrm{~K}$ versus $5 \times 10^{5} \mathrm{~K}$. Observation of such feature suggests that the HSS met and interacted in the heliosphere with one or several CMEs.

By analysis of the probable CMEs observed by STA, STB and LASCO in the period from 25 to 27 December 2011, we identified, as the probable solar sources, several CMEs starting in the period between 25 and 27 December. Three directed to the Earth brightest CMEs observed by STA, STB and LASCO are listed in Table 2. These CMEs were also traced in the heliosphere by the High resolution Imagers on STA and STB ${ }^{10}$. DBM predicted that these CMEs taken separately might arrive to the Earth in the period from 28 December 2011, 13:00 UT to 30 December 2011, 06:00 UT with the speeds $390-420 \mathrm{~km} \mathrm{~s}^{-1}$. Figure 6 shows a snapshot of the WSA-ENLIL Cone simulation of the solar wind conditions at the Earth on 29 December 2011, 00:00 UT, in the ecliptic and meridional planes. It occurs that these CMEs merged, created the complex ejecta and interacted with the faster HSS, which overtook them in the heliosphere. The WSA-ENLIL Cone simulation also shows that the main flow of the HSS propagated southward from the ecliptic plane, and only its small northern flank (in the meridional plane) moved in the direction to the Earth. We suppose that due to higher density and stronger magnetic field of the complex ejecta, its interaction with the HSS flank resulted in creation of a stratified structure with mixed HSS and CME plasma moving with the speed of the densest component - the slow CME complex. Probably, this effect might be caused by the physical mechanism as in the case of interaction of two CMEs described by Temmer et al. (2012), in which the fastest CME was decelerated by the slowest one.

Agreement of the measured HSS arrival time with the predicted one suggests that this interaction occurred close to the Earth. After 2 January 2012, 04:00 UT, the HSS signatures in the solar wind changed for the signatures of the second

\footnotetext{
$\overline{10}$ https://www.helcats-fp7.eu/catalogues/wp3_cat.html
} 

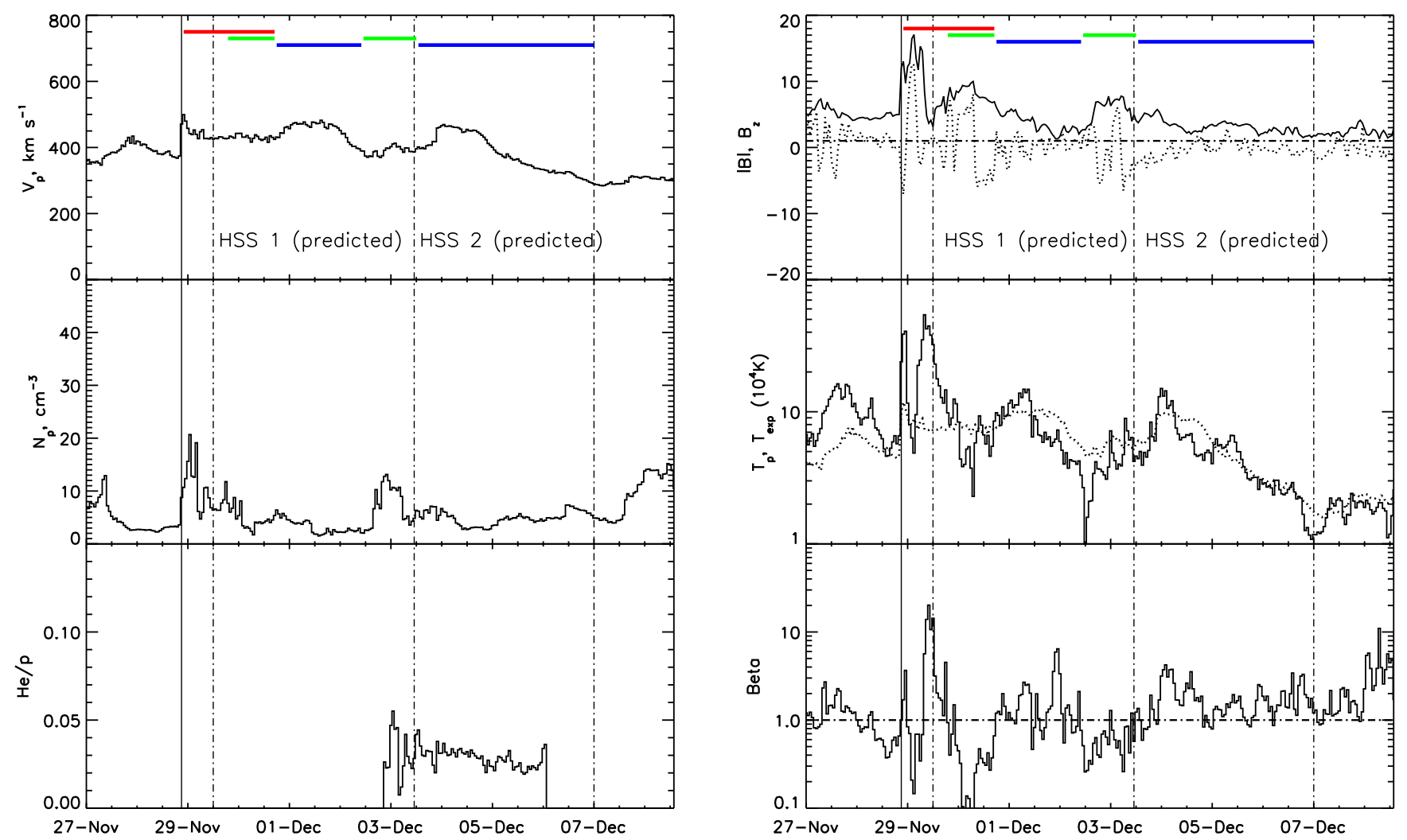

Fig. 3. Solar wind parameters for the period 27 November 2011, 00:00 UT-08 December 2012, 12:00 UT (CR 2117). The dash-dotted lines implicate the predicted period of the HSS arrival to the Earth. The dotted lines correspond to: in the proton temperature graph - the expected temperature, in the magnetic field graph $-B_{z}$ component. The solid vertical line shows a shock. The solar wind phenomena marked by the color bars are: HSSs (blue bar), ICMEs (green bar), CME-HSS interaction regions (red bar).

ICME probably produced by the CME started on 29 December 2011 at 17:24 UT (STA). This CME overlaid the HSS tail in the heliosphere arriving to the Earth with the speed $358 \mathrm{~km} \mathrm{~s}^{-1}$, which well agreed with the predicted by DBM value of $400 \mathrm{~km} \mathrm{~s}^{-1}$ in Table 2 .

At CR 2119 (Fig. 7), the HSS was predicted during the period from 27 January 2012, 05:00 UT to 31 January 2012, 12:00 UT with the speed $565 \mathrm{~km} \mathrm{~s}^{-1}$. A day earlier, on 23 January 2012 about 04:00 UT a fast CME started with the speed exceeding $1000 \mathrm{~km} \mathrm{~s}^{-1}$, which was predicted by DBM to arrive on 25 January at 17:00 UT with the speed of $480 \mathrm{~km} \mathrm{~s}^{-1}$. In contrary with the previous rotations, at CR 2119 the HSSCME interaction was not seen in the solar wind, so the arrival time and maximum speed of HSS predicted by the HE model agreed with the measurements. In the end of the HSS period, on 30 January 2012, 16:00 UT, WIND observed a fast forward shock in the solar wind (see the Heliospheric Shock Database $^{11}$ ). Analysis of the CME events at the Sun in the end of January 2012 has shown that STA and STB did not registered any CMEs directed to the Earth, which could produce this shock. However, on 27 January 2012 at 18:27 UT, LASCO registered near the western limb a halo CME with the speed $2500 \mathrm{~km} \mathrm{~s}^{-1}$, which was associated with the X-ray flare $\mathrm{X} 1.7$ observed by GOES. Although this powerful CME propagated towards STA in the direction perpendicular to the

11 http://ipsocks.fi
Sun-Earth line, it produced the global shock detected by WIND without the ICME signatures afterwards.

\section{Summary}

We present a case study of significant disagreement between the predicted and observed parameters of HSSs of solar wind revealed in CR 2118 - the second of three consecutive appearances of the recurrent $\mathrm{CH}$ in November 2011- February 2012 (CRs 2117-2119), when propagation of the HSSs in the heliosphere were accompanied by CMEs on the Sun. For prediction, we used two models. The first one is the kinematical hierarchical empirical model (HE model: Shugay et al., 2011, 2014) based on a relation between the HSS speed and area of the $\mathrm{CH}$ in the central part of the solar disk measured from the SDO/AIA images in the $19.3 \mathrm{~nm}$ band. The second is the WSA-ENLIL Cone model (Mays et al., 2015), which uses as the initial data for the HSS prediction the magnetic field expansion factor dependent on the position and area of the open magnetic field, and for the CME prediction the coronagraph data. Both models predict an increase of the HSS speed with the $\mathrm{CR}$ number due to the growing $\mathrm{CH}$ area in the central part of the solar disk, not taking into account probable interaction between the HSSs and the CMEs simultaneously propagated in the heliosphere.

From analysis of the solar wind parameters measured insitu by ACE and WIND, we found that at CR 2117 and CR 
2011-11-29T02:00

(a) Ecliptic plane

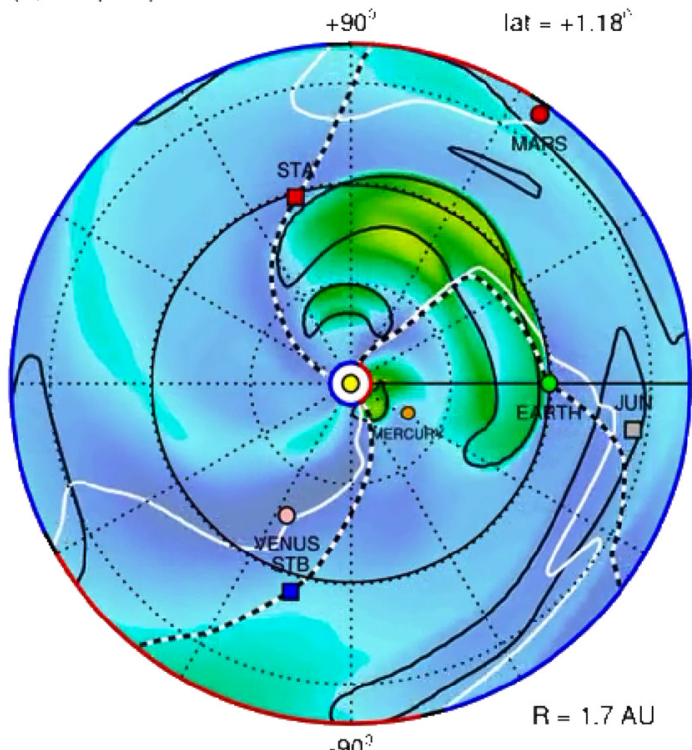

$\operatorname{Vr}(\mathrm{km} / \mathrm{s})$

$200 \quad 400 \quad 600 \quad 800 \quad 1000 \quad 1200 \quad 1400 \quad 1600$

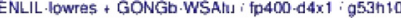

\section{EARTH}

(b) Meridional plane

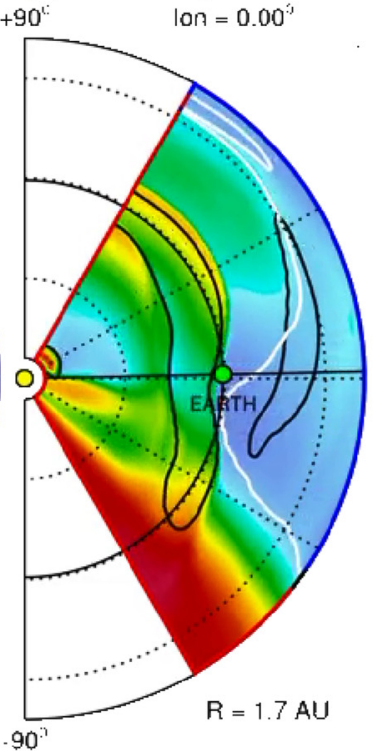

CME IMF line IMF polarity

Fig. 4. Snapshot of the WSA-ENLIL Cone simulation of the solar wind conditions in the heliosphere on 29 November 2011, 02:00 UT in the ecliptic plane (a) and meridional plane (b) (http://helioweather.net).
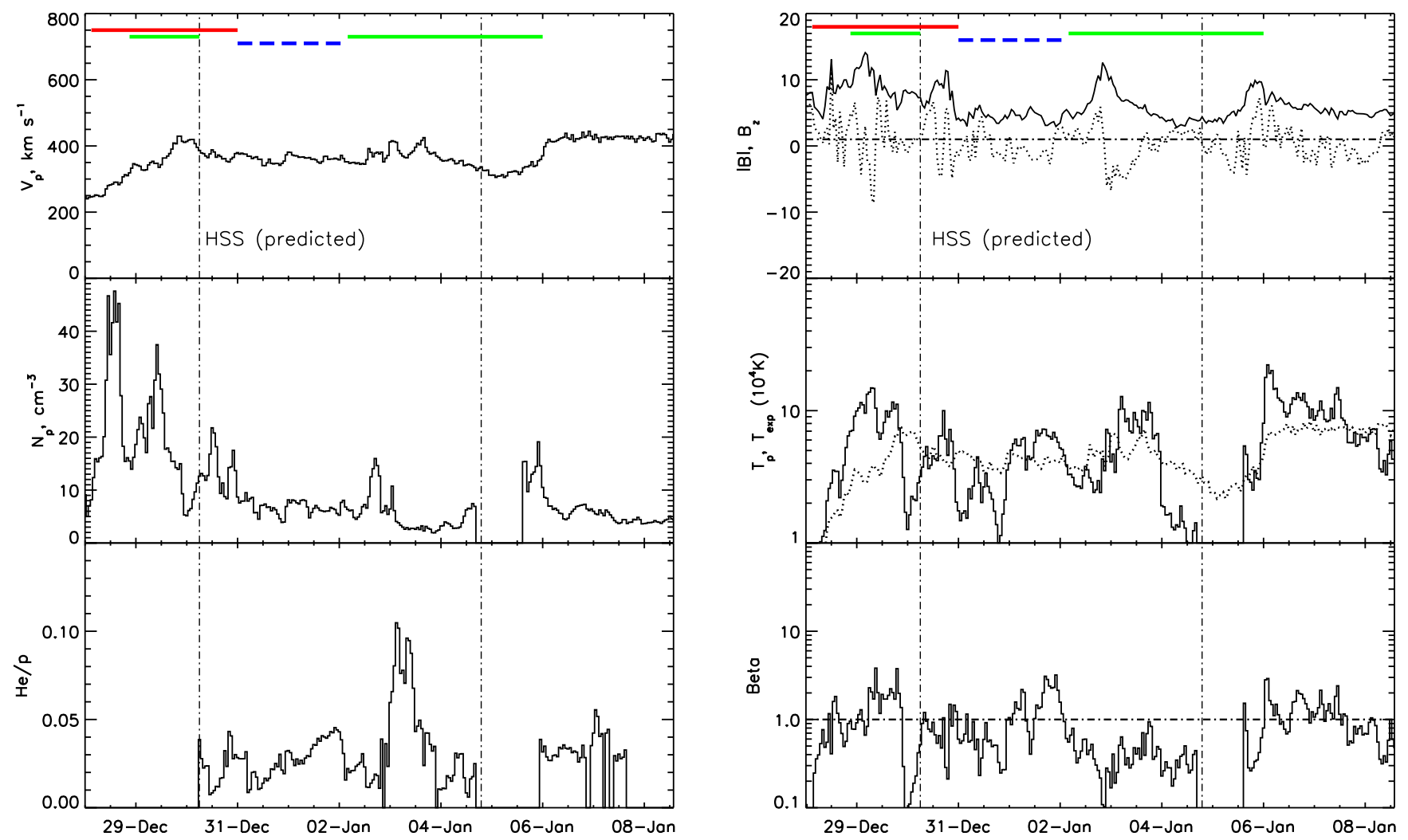

Fig. 5. The same as in Figure 3, for the interval 28 December 2011, 00:00 UT-08 January 2012, 12:00 UT (CR 2118). 
2011-12-29T00:00

(a) Ecliptic plane

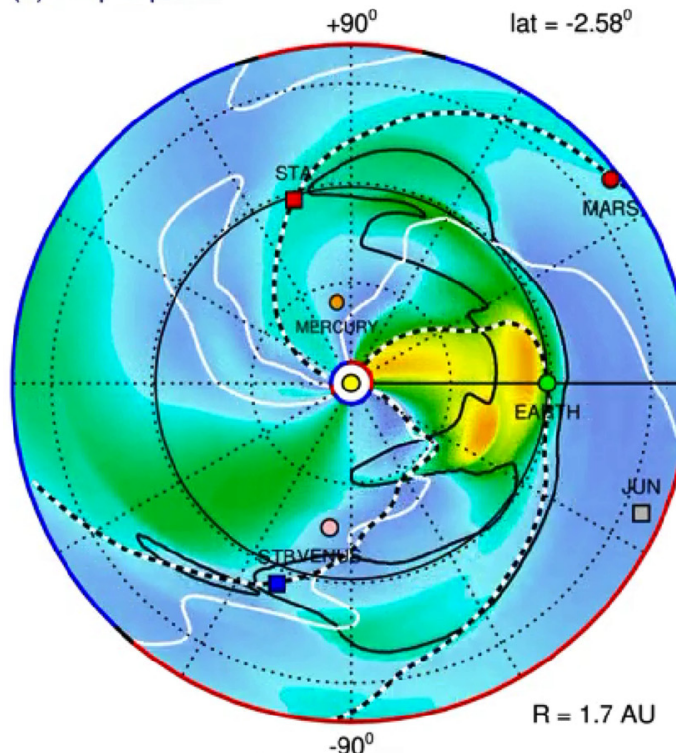

$\operatorname{Vr}(\mathrm{km} / \mathrm{s})$

$\begin{array}{llllllll}200 & 400 & 600 & 800 & 1000 & 1200 & 1400 & 1600\end{array}$

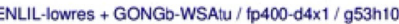

EARTH

(b) Meridional plane

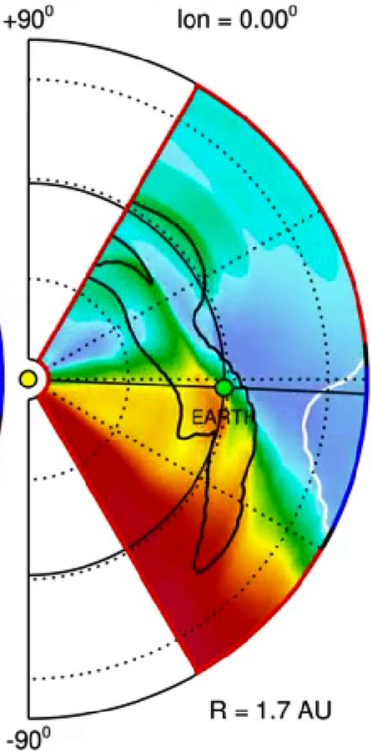

CME IMF line IMF polarity

Fig. 6. Snapshot of the WSA-ENLIL Cone simulation of the solar wind conditions in the heliosphere on 29 December 2011, 00:00 UT in the ecliptic plane (a) and meridional plane (b).
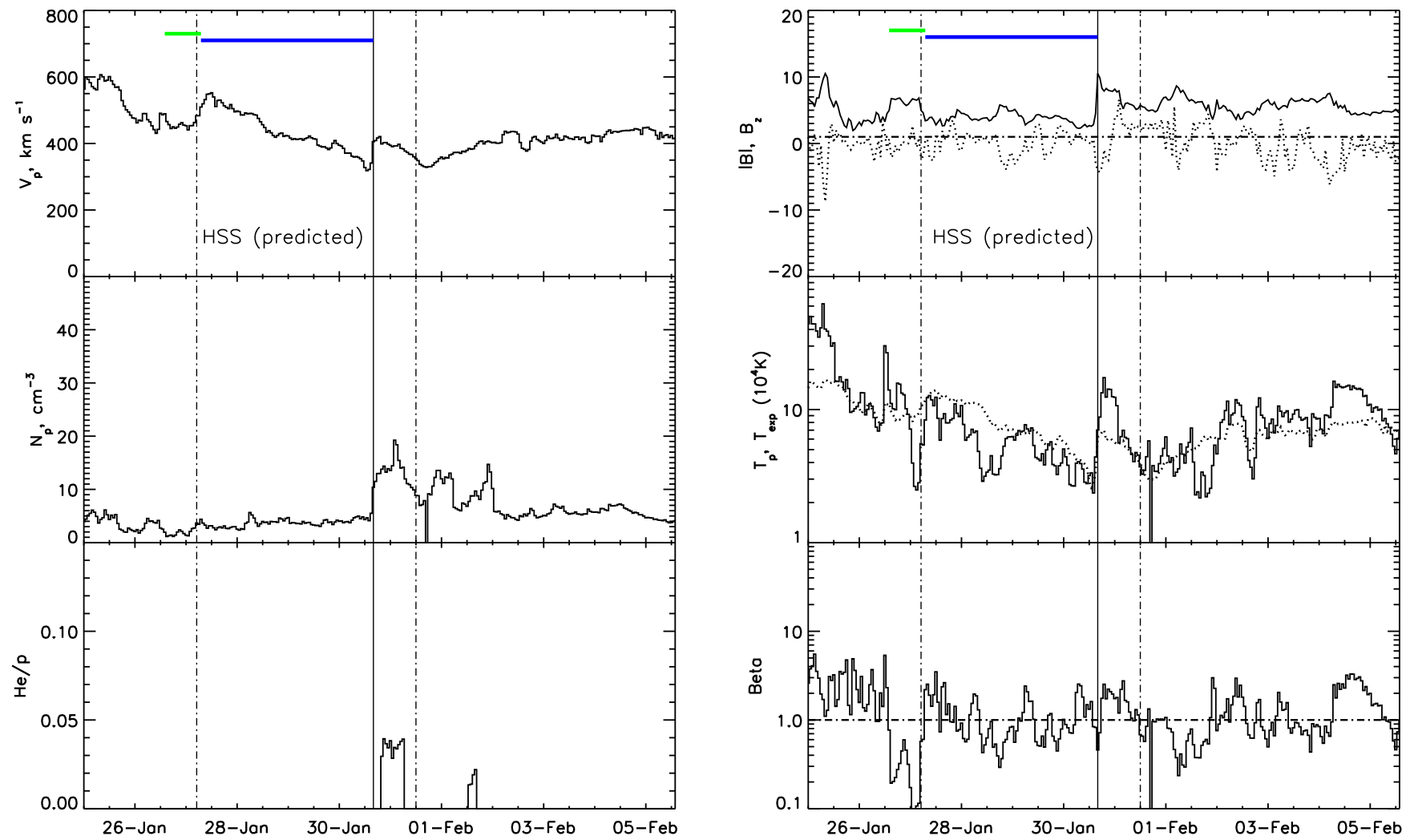

Fig. 7. The same as in Figure 3, for the interval 25 January 2012, 00:00 UT-05 February 2012, 12:00 UT (CR 2119). 
2119 the measured speeds were close to the predicted values with the difference of $57 \mathrm{~km} \mathrm{~s}^{-1}$ and $82 \mathrm{~km} \mathrm{~s}^{-1}$. At CR 2118 , instead, the measured HSS speed was on $217 \mathrm{~km} \mathrm{~s}^{-1}$ less than the predicted value. We suppose that the low speed of the solar wind in the place of predicted HSS at CR 2118 might be caused by interaction with the complex of CMEs propagated at the same period in the heliosphere. By analysis of the events presented in the STEREO and LASCO CME lists, we selected the CMEs propagated towards the Earth, which might interact with the HSSs during their passage in the heliosphere.

We found that at CR 2117, nine hours after the slow HSS ( $V \sim 425 \mathrm{~km} \mathrm{~s}^{-1}$, a fast CME started with the speed of $\sim 1000 \mathrm{~km} \mathrm{~s}^{-1}$, overtook the HSS near the Earth, and the both streams arrived to the Earth simultaneously with similar speeds of about $450 \mathrm{~km} \mathrm{~s}^{-1}$. Because of this interaction, the HSS signatures in the solar wind appeared later than the predicted arrival time, but due to similarity of the stream speeds, the HSS speed was very close to the predicted value.

At CR 2118, instead, the faster HSS overtook the slower moving complex ejecta originated from the series of merged CMEs, which arrived to the Earth just before the HSS. We suggest that due to its higher density and magnetic field strength, the complex ejecta represented an obstacle for the HSS and slowed down its equatorial flank on $217 \mathrm{~km} \mathrm{~s}^{-1}$.

At CR 2119, the closest in time CME directed to the Earth arrived ahead of the HSS. In contrary with the previous rotations, at CR 2119, the signatures of the HSS-CME interaction were absent, so the HSS speed predicted by the HE model agrees with the solar wind measurements within the typical error of $\pm 100 \mathrm{~km} \mathrm{~s}^{-1}$.

In conclusion, our study shows the case, when the HSSCME interaction in the heliosphere affected on the HSS propagation, which resulted in disagreement between the HSS parameters measured in-situ and predicted by the models based on the $\mathrm{CH}$ geometry. The effect of such interaction depends on the 3D geometry and propagation directions of the HSS and CMEs as well as on the relations between their timing and speeds. Probably, this interaction has similar physical nature as in the case of deceleration of the fast CME by the slower one described by Temmer et al. (2012). We suggest that significant disagreement between predictions and measurements of HSSs in-situ at $1 \mathrm{AU}$ exceeding the accuracy of the used model may serve as an indicator of strong HSS-CME interaction for further investigation.

Acknowledgements. The authors are grateful for the opportunity to use data of the SDO/AIA telescope ${ }^{12}$. We have used the CME catalog that is generated and maintained at the CDAW Data Center ${ }^{13}$ by NASA and The Catholic University of America in cooperation with the Naval Research Laboratory. $\mathrm{SOHO}$ is a project of international cooperation between ESA and NASA. Also this paper uses data from the SEEDS CME catalog $^{14}$. The SEEDS project has been supported by NASA Living With a Star Program and NASA Applied Information

\footnotetext{
$\overline{12}$ http://sdo.gsfc.nasa.gov

$13 \mathrm{https} / / /$ cdaw.gsfc.nasa.gov/

$14 \mathrm{http}: / /$ spaceweather.gmu.edu/seeds/

$15 \mathrm{http}: / /$ helioweather.net

$16 \mathrm{http} / / /$ oh.geof.unizg.hr/DBM/dbm.php

$17 \mathrm{http} / / /$ solardemon.oma.be/
}

Systems Research Program. The OMNI data were obtained from the GSFC/SPDF OMNIWeb interface at https://omni web.gsfc.nasa.gov. We are grateful for the opportunity to use the results of the simulation obtained by the WSA-Enlil Cone ${ }^{15}$ and DBM ${ }^{16}$ models. The authors thank the STEREO, GOES, WIND, and ACE research teams for their open data policy. We have used the Solar Demon database ${ }^{17}$ that has been constructed under the AFFECTS and COMESEP projects of the EC FP7 Programme. This work was supported by the Russian Scientific Foundation projects: 16-17-00098 (Yu. Shugay, prediction of the HSSs and comparison with measurements, sections 2.1, 3.1); 17-12-01567 (V. Slemzin, D. Rodkin, identification of the solar wind phenomena in-situ and their solar sources, sections 2.2, 3.2). The editor thanks two anonymous referees for their assistance in evaluating this paper.

\section{References}

Alves MV, Echer E, Gonzalez WD. 2006. Geoeffectiveness of corotating interaction regions as measured by Dst index. J Geophys Res (Space Phys) 111: A07S05. DOI:10.1029/2005JA011379.

Arge CN, Pizzo VJ. 2000. Improvement in the prediction of solar wind conditions using near-real time solar magnetic field updates. $J$ Geophys Res (Space Phys) 105: 10465-10480. DOI:10.1029/ 1999JA000262.

Behannon KW, Hewish A, Burlaga LF. 1991. Structure and evolution of compound streams at not greater than $1 \mathrm{AU} . J$ Geophys Res 96: 21. DOI:10.1029/91JA02267.

Borovsky JE, Denton MH. 2010. Solar wind turbulence and shear: a superposed-epoch analysis of corotating interaction regions at 1 AU. J Geophys Res (Space Phys) 115: A10101. DOI:10.1029/ 2009JA014966.

Brueckner GE, Howard RA, Koomen MJ, Korendyke CM, Michels DJ, et al. 1995. The large angle spectroscopic coronaXXX (LASCO). Sol Phys 162: 357-402. DOI:10.1007/BF00733434.

Burlaga LFE. 1991. Magnetic clouds 152.

Burlaga LF, Plunkett SP, St Cyr OC. 2002. Successive CMEs and complex ejecta. J Geophys Res (Space Phys) 107: 1266. DOI:10.1029/2001JA000255.

Burlaga L, Berdichevsky D, Gopalswamy N, Lepping R, Zurbuchen T. 2003. Merged interaction regions at 1 AU. J Geophys Res (Space Phys) 108: 1425. DOI:10.1029/2003JA010088.

Domingo V, Fleck B, Poland AI. 1995. The SOHO mission: an overview. Sol Phys 162: 1-37. DOI:10.1007/BF00733425.

Echer E, Tsurutani BT, Gonzalez WD, Kozyra JU. 2011. High speed stream properties and related geomagnetic activity during the whole heliosphere interval (WHI): 20 March-16 April 2008. Sol Phys 274: 303-320. DOI:10.1007/s11207-011-9739-0.

Fenrich FR, Luhmann JG. 1998. Geomagnetic response to magnetic clouds of different polarity. Geophys Res Lett 25: 2999-3002. DOI:10.1029/98GL51180.

Gloeckler G, Balsiger H, Bürgi A, Bochsler P, Fisk LA, et al. 1995. The solar wind and suprathermal ion composition investigation on the WIND spacecraft. Space Sci Rev 71: 79-124. DOI:10.1007/ BF00751327.

Gopalswamy N, Yashiro S, Krucker S, Stenborg G, Howard RA. 2004. Intensity variation of large solar energetic particle events associated with coronal mass ejections. J Geophys Res (Space Phys) 109: A12105. DOI:10.1029/2004JA010602. 
Gopalswamy N, Mäkelä P, Xie H, Akiyama S, Yashiro S. 2009. CME interactions with coronal holes and their interplanetary consequences. J Geophys Res (Space Phys) 114: A00A22. DOI:10.1029/2008JA013686.

Harvey JW, Sheeley NR. 1978. Coronal holes, solar wind streams, and geomagnetic activity during the new sunspot cycle. Sol Phys 59: 159-173. DOI:10.1007/BF00154939.

Howard RA, Moses JD, Vourlidas A, Newmark JS, Socker DG, et al. 2008. Sun Earth connection coronal and heliospheric investigation (SECCHI). Space Sci Rev 136: 67-115. DOI:10.1007/ s11214-008-9341-4.

Hurlburt N, Cheung M, Schrijver C, Chang L, Freeland S, et al. 2012. Heliophysics event knowledgebase for the Solar Dynamics Observatory (SDO) and beyond. Sol Phys 275: 67-78. DOI:10.1007/s11207-010-9624-2.

Jian LK, MacNeice PJ, Taktakishvili A, Odstrcil D, Jackson B, Yu HS, Riley P, Sokolov IV, Evans RM. 2015. Validation for solar wind prediction at Earth: comparison of coronal and heliospheric models installed at the CCMC. Space Weather 13: 316-338. DOI: 10.1002/ 2015 SW001174.

Kilpua, EKJ, Hietala H, Turner DL, Koskinen HEJ, Pulkkinen TI, Rodriguez JV, Reeves GD, Claudepierre SG, Spence HE. 2015. Unraveling the drivers of the storm time radiation belt response. Geophys Res Lett 42: 3076-3084. DOI:10.1002/2015GL063542.

Krista LD, Gallagher PT. 2009. automated coronal hole detection using local intensity thresholding techniques. Sol Phys 256: 87100. DOI:10.1007/s11207-009-9357-2.

Lemen JR, Title AM, Akin DJ, Boerner PF, Chou C, et al. 2012. The Atmospheric Imaging Assembly (AIA) on the Solar Dynamics Observatory (SDO). Sol Phys 275: 17-40. DOI:10.1007/ s11207-011-9776-8.

Lepping RP, Berdichevsky DB, Szabo A, Arqueros C, Lazarus AJ. 2003. Profile of an average magnetic cloud at $1 \mathrm{AU}$ for the quiet solar phase: wind observations. Sol Phys 212: 425-444. DOI:10.1023/A:1022938903870.

Mays ML, Taktakishvili A, Pulkkinen A, MacNeice PJ, Rastätter L, et al. 2015. Ensemble modeling of CMEs using the WSAENLIL+Cone model. Sol Phys 290: 1775-1814. DOI:10.1007/ s11207-015-0692-1.

McComas DJ, Bame SJ, Barker P, Feldman WC, Phillips JL, Riley P, Griffee JW. 1998. Solar wind electron proton alpha monitor (SWEPAM) for the Advanced Composition Explorer. Space Sci Rev 86: 563-612. DOI:10.1023/A:1005040232597.

Mishra W, Srivastava N, Chakrabarty D. 2015. Evolution and consequences of interacting CMEs of 9-10 November 2012 Using STEREO/SECCHI and In Situ observations. Sol Phys 290: 527552. DOI:10.1007/s11207-014-0625-4.

Mitsakou E, Moussas X. 2014. Statistical study of ICMEs and their sheaths during solar cycle 23 (1996-2008). Sol Phys 289: $3137-$ 3157. DOI:10.1007/s11207-014-0505-y.

Mohamed AA, Gopalswamy N, Yashiro S, Akiyama S, Mäkelä P, Xie H, Jung H. 2012. The relation between coronal holes and coronal mass ejections during the rise, maximum, and declining phases of solar cycle 23. J Geophys Res (Space Phys) 117: A01103. DOI:10.1029/2011JA016589.

Möstl C, Rollett T, Frahm RA, Liu YD, Long DM, et al. 2015. Strong coronal channelling and interplanetary evolution of a solar storm up to Earth and Mars. Nat Commun 6: 7135. DOI:10.1038/ ncomms 8135 .

Myagkova IN, Shugay YS, Veselovsky IS, Yakovchouk OS. 2013. Comparative analysis of recurrent high-speed solar wind streams influence on the radiation environment of near-earth space in April-
July 20101. Sol Syst Res 47: 127-140. DOI:10.1134/ S0038094613020068.

Nieves-Chinchilla T, Vourlidas A, Stenborg G, Savani NP, Koval A, Szabo A, Jian LK. 2013. Inner heliospheric evolution of a "Stealth" CME derived from multi-view imaging and multipoint in situ observations. I. Propagation to 1 AU. Astrophys J 779: 55. DOI:10.1088/0004-637X/779/1/55.

Nolte JT, Krieger AS, Timothy AF, Gold RE, Roelof EC, Vaiana G, Lazarus AJ, Sullivan JD, McIntosh PS. 1976. Coronal holes as sources of solar wind. Sol Phys 46: 303-322. DOI:10.1007/ BF00149859.

Odstrčil D, Pizzo VJ. 1999. Three-dimensional propagation of CMEs in a structured solar wind flow: 1. CME launched within the streamer belt. J Geophys Res 104: 483-492. DOI:10.1029/ 1998JA900019.

Pizzo V, Millward G, Parsons A, Biesecker D, Hill S, Odstrcil D. 2011. Wang-Sheeley-Arege-ENLIL cone model transitions to operations. Space Weather 9: S03004. DOI:10.1029/ 2011SW000663.

Prise AJ, Harra LK, Matthews SA, Arridge CS, Achilleos N. 2015. Analysis of a coronal mass ejection and corotating interaction region as they travel from the Sun passing Venus, Earth, Mars, and Saturn. J Geophys Res (Space Phys) 120: 1566-1588. DOI:10.1002/2014JA020256.

Reiss MA, Temmer M, Veronig AM, Nikolic L, Vennerstrom S, Schöngassner F, Hofmeister SJ. 2016. Verification of high-speed solar wind stream forecasts using operational solar wind models. Space Weather 14: 495-510. DOI:10.1002/2016SW001390.

Rod'kin DG, Shugay YS, Slemzin VA, Veselovskii IS. 2016. The effect of solar activity on the evolution of solar wind parameters during the rise of the 24th cycle. Sol Syst Res 50: 44-55. DOI:10.1134/S0038094616010032.

Rotter T, Veronig AM, Temmer M, Vršnak B. 2015. Real-time solar wind prediction based on SDO/AIA coronal hole data. Sol Phys 290: 1355-1370. DOI:10.1007/s11207-015-0680-5.

Rouillard AP, Lavraud B, Sheeley NR, Davies JA, Burlaga LF, Savani NP, Jacquey C, Forsyth RJ. 2010. White light and in situ comparison of a forming merged interaction region. Astrophys $J$ 719: 1385-1392. DOI: 10.1088/0004-637X/719/2/1385.

Scholl IF, Habbal SR. 2008. Automatic detection and classification of coronal holes and filaments based on EUV and magnetogram observations of the solar disk. Sol Phys 248: 425-439. DOI:10.1007/s11207-007-9075-6.

Sheeley NR Jr., Harvey JW. 1981. Coronal holes, solar wind streams, and geomagnetic disturbances during 1978 and 1979. Sol Phys 70: 237-249. DOI:10.1007/BF00151331.

Shugay YS, Veselovsky IS, Seaton DB, Berghmans D. 2011. Hierarchical approach to forecasting recurrent solar wind streams. Sol Syst Res 45: 546-556. DOI:10.1134/S0038094611060086.

Shugay Y, Slemzin V, Veselovsky I. 2014. Magnetic field sector structure and origins of solar wind streams in 2012. J Space Weather Space Clim 4: A24. DOI:10.1051/swsc/2014021.

Shugay YS, Veselovsky D, Slemzin VA, Yermolaev YI, Rodkin DG. 2017. Possible causes of the discrepancy between the predicted and observed parameters of high-speed solar wind streams. Cosm Res 55: 20-29. DOI:10.1134/S0010952517010087.

Slemzin VA, Shugai YS. 2015. Identification of coronal sources of the solar wind from solar images in the EUV spectral range. Cosm Res 53: 47-58. DOI:10.1134/S0010952515010074.

Smith CW, L'Heureux J, Ness NF, Acuña MH, Burlaga LF, Scheifele J. 1998. The ACE magnetic fields experiment. Space Sci Rev 86: 613-632. DOI:10.1023/A:1005092216668. 
Tsurutani BT, Echer E, Gonzalez WD. 2011. The solar and interplanetary causes of the recent minimum in geomagnetic activity (MGA23): a combination of midlatitude small coronal holes, low IMF $\mathrm{B}_{\mathrm{Z}}$ variances, low solar wind speeds and low solar magnetic fields. Ann Geophys 29: 839-849. DOI:10.5194/angeo29-839-2011.

Temmer M, Vršnak B, Rollett T, Bein B, de Koning CA, et al. 2012. Characteristics of kinematics of a coronal mass ejection during the 2010 August 1 CME-CME interaction event. Astrophys J 749: 57. DOI:10.1088/0004-637X/749/1/57.

Temmer M, Reiss MA, Nikolic L, Hofmeister SJ, Veronig AM. 2017. Preconditioning of interplanetary space due to transient CME disturbances. Astrophys $J$ 835: 141. DOI:10.3847/1538-4357/835/ 2/141.

Tsurutani BT, Gonzalez WD, Gonzalez ALC, Tang F, Arballo JK, Okada M. 1995. Interplanetary origin of geomagnetic activity in the declining phase of the solar cycle. J Geophys Res 100: 21717 21734. DOI: $10.1029 / 95 J A 01476$.

Tsurutani BT, Gonzalez WD, Gonzalez ALC, Guarnieri FL, Gopalswamy N, et al. 2006. Corotating solar wind streams and recurrent geomagnetic activity: a review. $J$ Geophys Res (Space Phys) 111: A07S01. DOI:10.1029/2005JA011273.

Tsurutani BT, Echer E, Gonzalez WD. 2011a. The solar and interplanetary causes of the recent minimum in geomagnetic activity (MGA23): a combination of midlatitude small coronal holes, low IMF $B_{Z}$ variances, low solar wind speeds and low solar magnetic fields. Annales Geophysicae 29: 839-849. DOI:10.5194/ angeo-29-839-2011.

Tsurutani BT, Echer E, Guarnieri FL, Gonzalez WD. 2011b. The properties of two solar wind high speed streams and related geomagnetic activity during the declining phase of solar cycle $23 . J$ Atmos Sol-Terr Phys 73: 164-177. DOI:10.1016/j. jastp.2010.04.003.
Vršnak B, Temmer M, Veronig AM. 2007. Coronal holes and solar wind high-speed streams: I. forecasting the solar wind parameters. Sol Phys 240: 315-330. DOI:10.1007/s11207-007-0285-8.

Vršnak B, Žic T, Vrbanec D, Temmer M, Rollett T, et al. 2013. Propagation of interplanetary coronal mass ejections: the dragbased model. Sol Phys 285: 295-315. DOI:10.1007/ s11207-012-0035-4.

Wang Y-M, Sheeley Jr NR. 1990. Solar wind speed and coronal fluxtube expansion. Astrophys J 355: 726-732. DOI:10.1086/168805.

Yermolaev YI, Yermolaev MY, Zastenker GN, Zelenyi LM, Petrukovich AA, Sauvaud J-A. 2005. Statistical studies of geomagnetic storm dependencies on solar and interplanetary events: a review. Planet Space Sci 53: 189-196. DOI:10.1016/j. pss.2004.09.044.

Yermolaev YI, Nikolaeva NS, Lodkina IG, Yermolaev MY. 2009. Catalog of large-scale solar wind phenomena during 1976-2000. Cosm Res 47: 81-94. DOI:10.1134/S0010952509020014.

Yermolaev YI, Nikolaeva NS, Lodkina IG, Yermolaev MY. 2010. Specific interplanetary conditions for CIR-, Sheath-, and ICMEinduced geomagnetic storms obtained by double superposed epoch analysis. Ann Geophys 28: 2177-2186. DOI:10.5194/angeo28-2177-2010.

Yermolaev YI, Lodkina IG, Nikolaeva NS, Yermolaev MY. 2015. Dynamics of large-scale solar wind streams obtained by the double superposed epoch analysis. J Geophys Res (Space Phys) 120: 7094-7106. DOI:10.1002/2015JA021274.

Yokoyama N, Kamide Y. 1997. Statistical nature of geomagnetic storms. J Geophys Res 102: 14215-14222. DOI:10.1029/ 97JA00903.

Zhao X, Dryer M. 2014. Current status of CME/shock arrival time prediction. Space Weather 12: 448-469. DOI: 10.1002/ 2014SW001060.

Zurbuchen TH, Richardson IG. 2006. In-situ solar wind and magnetic field signatures of interplanetary coronal mass ejections. Space Sci Rev 123: 31-43. DOI: 10.1007/s11214-006-9010-4.

Cite this article as: Shugay Y, Slemzin V, Rodkin D, Yermolaev Y, Veselovsky I. 2018. Influence of coronal mass ejections on parameters of high-speed solar wind: a case study. J. Space Weather Space Clim. 8: A28 